\title{
Multi-walled carbon nanotube sensor coupled with a pre- concentrator nanoporous layer for the detection of benzene at low temperature
}

\author{
Frank James ${ }^{1}$, Dominique Porterat ${ }^{1}$, Farhad Abedini², Martine Mayne-L'Hermite' ${ }^{1}$, Thu-hoa Tran-Thi' \\ ${ }^{1}$ NIMBE, CEA, CNRS, Université Paris-Saclay 91191 Gif-sur-Yvette, France, \\ ${ }^{2}$ ETHERA, 628 rue Charles de Gaulle, 38920 Crolles, France \\ Corresponding author's e-mail address: martine.mayne@cea.fr,
}

\begin{abstract}
This work reports the development and study of a resistive gas sensor for benzene detection, based on multi-walled carbon nanotubes (MWCNTs). The MWCNTs were covered with a nanoporous $\mathrm{SiO}_{2}$ layer whose function is to concentrate the pollutant in order to enhance the sensor performances. In this paper, we will describe the preparation of the sensor and highlight the beneficial effects of both the preconcentration layer and the operating temperature. MWCNT/SiO 2 -based sensors operating at $125^{\circ} \mathrm{C}$ are able to detect $50 \mathrm{ppb}$ of benzene in air. Further developments are in progress in order to improve this detection limit.
\end{abstract}

Key words: gas sensor, carbon nanotube-based sensor, benzene, concentrator layer, low temperature.

\section{Introduction}

There is a high demand for compact solid-state and low cost sensors to detect monocyclic aromatic hydrocarbons in the sub-ppm range at low temperature. Over the years, a great effort has been made to use carbon nanotubes (CNT) as gas sensing materials with high specific surface area. Several groups have demonstrated the great ability of CNT for detection of gases [1], especially when they are metal-decorated and/or chemically functionalized [2], which increases the sensitivity.

In this context, our objective is to design and optimize a chemical sensor based on multiwalled carbon nanotubes (MWCNTs) which sensitivity arises via coupling with a preconcentration nanoporous silica layer. The detection mechanism is based on the resistance change of the resistive CNT upon gas exposure. The resistance change is due to the p-type semiconducting behavior of the MWCNTs [3]. Our choice of nanoporous silica as preconcentrator for the sensor is based on a previous work which has shown the ability of various thick nanoporous silica matrices to trap benzene and toluene over the ppb to ppm range [4]. Here we will show that it is possible to use a thin layer of functionalized silica as preconcentrator to trap and concentrate benzene and toluene at the vicinity of CNT.

\section{Sensitive hybrid material}

The MWCNTs used for the fabrication of the sensor are synthesized via an aerosol- assisted chemical vapor deposition process [3]. Thermally treated MWCNTs were obtained by annealing raw MWCNTs at $2000^{\circ} \mathrm{C}$ under an argon atmosphere for one hour. This treatment induces the elimination of metal-based catalyst impurities in the MWCNTs and enhances the structural quality of raw MWCNTs [5].

The thin film of $\mathrm{SiO}_{2}$ coated on the MWCNTs is synthesized via the sol-gel process with two silicon alkoxide precursors, tetramethoxylane (TMOS) and phenyltetramethoxylane (PhTMOS) in methanol solvent. Water is then added to start the hydrolysis and condensation reaction. After 10 minutes of maturation, a drop of the mixture produced (Sol) is deposited on gold interdigitated electrodes where it became solid (Gel), to build the sensor device.

\section{Preparation of gas sensor and detection method}

The gas sensors fabricated with MWCNTs, shown in Fig.1, were qualified in terms of electrical resistance. The initial electrical resistance is in the [50-150 $\Omega$ ] range in order to get a suitable signal/noise ratio for the detection tests. The operating temperature is controlled through a power supply, with a multimeter used for the data acquisition. 


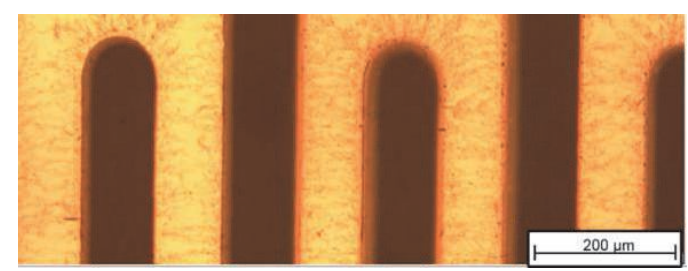

Fig. 1. Optical microscopy picture of MWCNTs deposited between electrodes

The target gas was injected into a dilution system. Before each exposure to a given benzene concentration, the sensor was first stabilized at the nominal heating voltage under a carrier gas (nitrogen or air) until the electrical resistance signal reached a constant level. The sensor was then exposed to benzene during 25 min, with the electrical resistance changes measured all along the benzene exposure. All tests were performed under a total flow rate of 300 sccm. The sensitivity, calculated according: (R-Ro) x 100/Ro, with $R_{0}$, and $R$ the resistance under carrier gas and benzene respectively, was measured versus time.

\section{Detection tests of benzene}

Fig. 2 shows the response at room temperature of the MWCNT sensor compared with that of MWCNT covered with the $\mathrm{SiO}_{2}$ preconcentration layer when both were exposed to $1 \mathrm{ppm}$ of benzene diluted with air. We can highlight the fact that the detection is improved with the concentrator filter, the sensitivity being 4 times higher than that of MWCNT alone.

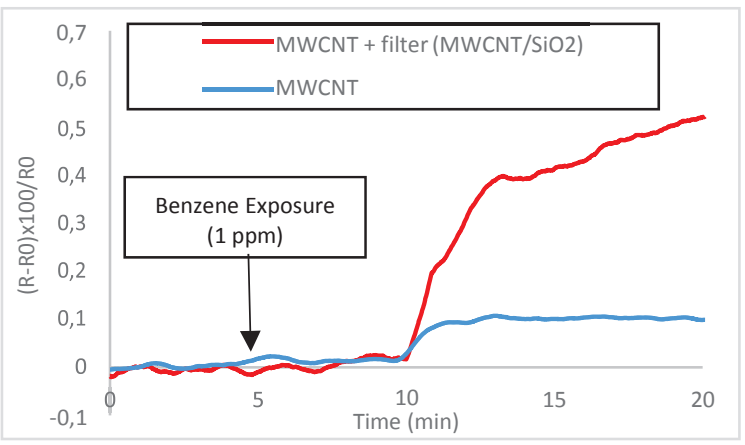

Fig. 2. Sensitivity comparison between MWCNT sensors with and without pre-concentration layer for 1 ppm benzene detection at room temperature

Nevertheless, the sensitivity values are still low (0.4\% maximum). Therefore, the sensors were heated in order to improve the detection.

Fig. 3 shows the sensitivity of both sensors exposed to a wide range of benzene concentrations (50 to $500 \mathrm{ppb}$ ). The optimized temperature, $125^{\circ} \mathrm{C}$, allows benzene detection at low concentration, e.g. $50 \mathrm{ppb}$ and at higher sensitivity as compared to room temperature. At $125^{\circ} \mathrm{C}$, the sensitivity and the detection limit are significantly improved for both sensors. In addition, the pre-concentration layer is quite efficient: the sensitivity is almost double than that of the MWCNT sensor.

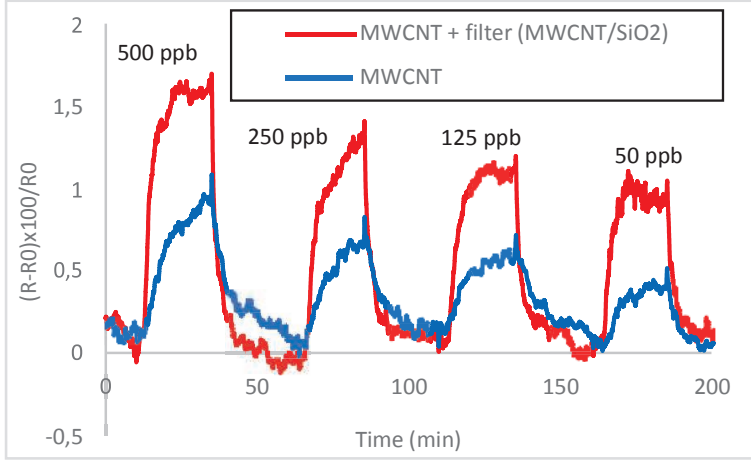

Fig. 3. Sensitivity of MWCNT sensors with and without pre-concentrator layer for 500, 250, 125 and 50 ppb of benzene operated at $125^{\circ} \mathrm{C}$

\section{Conclusion}

We have developed a sensor for the detection of benzene based on a novel concept of material by coupling multi-walled carbon nanotubes with a $\mathrm{SiO}_{2}$ pre-concentration layer. This sensor operating at $125^{\circ} \mathrm{C}$ can detect concentrations down to $50 \mathrm{ppb}$. These results underline the potential of this MWCNTs/SiO 2 hybrid material for the detection of indoor and outdoor air pollutants.

\section{Acknowledgments}

This work has been performed in the SMARTY framework. The authors thank BPI France for their financial support.

\section{References}

[1] D.R. Kauffman, A. Star, Carbon Nanotube Gas and Vapor Sensors, Angewandte Chemie International Edition 47 (2008) 6550-6570; doi: 10.1002/anie. 200704488

[2] I. V. Zaporotskovaa, N. P. Borozninaa , Y. N. Parkhomenkob , L. V. Kozhitovb, Carbon nanotubes: Sensor properties. A review, Modern Electronic Materials 2 (2016) 95-105; doi: org/10.1016/j.moem.2017.02.002

[3] A. Gohier, J. Chancolon, P.Chenevier, D. Porterat, M. Mayne-L'Hermite, C. Reynaud, Optimized network of multi-walled carbon nanotubes for chemical sensing, Nanotechnology 22 (2011); doi:105501

[4] L. Calvo-Muñoz, T-T. Truong, T-H. Tran-Thi, Chemical sensors of monocyclic aromatic hydrocarbons based on sol-gel materials: kinetics of trapping of the pollutants and sensitivity of the sensor, Sensors and Actuators B: Chemical 87 (2002) 173-183; 4005(02)00236-8 doi: 10.1016/S0925-

5] M. Pinault, V. Pichot, H. Khodja, P. Launois, C.c. Reynaud, M. Mayne-L'Hermite, Evidence of sequential lift in growth of aligned multiwalled carbon nanotube multilayers, Nano Letters 5 (2005) 2394-2398; doi: 10.1021/nl051472k 